\title{
Evaluación de los Frameworks en el Desarrollo de Aplicaciones Web con Python
}

\author{
Jimmy Rolando Molina Ríos, Nancy Magaly Loja Mora, Mariuxi Paola Zea Ordóñez, Erika Lizbeth Loaiza Sojos \\ Departamento de Investigación \\ Universidad Técnica de Machala \\ Machala, Ecuador \\ \{jmolina, nmloja, mzea, elloaiza_est\}@utmachala.edu.ec
}

\begin{abstract}
RESUMEN-Debido a la creciente interacción de los usuarios con sistemas web, surge la necesidad de combinar las funcionalidades de aplicaciones clásicas de escritorio, con la accesibilidad y bajo costo de la publicación de aplicaciones web; dando origen a la elección del mejor marco de trabajo que se adopte a las necesidades de los desarrolladores. Esta investigación presenta un análisis comparativo de los frameworks que trabajan con el lenguaje Python para el desarrollo de aplicaciones web. Para ello el análisis se formuló mediante un modelo de evaluación que se basa en las características de calidad propuestas en la norma ISO/IEC 9126. Estas a su vez permiten establecer sub-características, atributos y métricas para evaluar la calidad de las aplicaciones web. Permitiendo obtener como resultado una matriz para la Evaluación de Frameworks: Django, Pyramid, Turbogear y Web2PY. Los resultados obtenidos mostraron las fortalezas $y$ debilidades de cada framework y fue la base para determinar que Django es el mejor framework para la implementación de desarrollo de sistemas web. Este framework cumplió con todos los indicadores del modelo de evaluación, los resultados redactados al final del documento determinan que tomando en cuenta las métricas de calidad se puede elegir qué marco de trabajo es el que mejor se adapta para el desarrollo de aplicaciones web en la Ciudad de Machala. Antes de realizar una evaluación se considera indispensable conocer y comprender el funcionamiento de los elementos que se vaya a cotejar, para ello es recomendable emplear tablas para la comparación de las características, teniendo como referencia sitios web confiables que aporten documentación sobre los frameworks y el empleo de estándares de calidad para su determinación.
\end{abstract}

PALABRAS CLAVE-Aplicaciones web, frameworks, ISO/IEC 9126, modelo de evaluación.

\section{INTRODUCCIÓN}

En los últimos tiempos, el internet ha tenido un auge espectacular, se puede afirmar que la tecnología web ha demostrado ser considerablemente simple y flexible para publicar y encontrar información pertinente para el usuario, tanto así que en la actualidad la presencia de aplicaciones web es cada vez más evidente en empresas que sienten la necesidad de llegar a sus clientes con sus productos sin tener que ser desplazados por la competencia que existe en el mercado y a su vez buscando eficiencia en sus servicios $\mathrm{y}$ ofreciendo productos cada vez mejores y al mismo tiempo con mejores ofertas para así ganar marketing y mejores oportunidades.

Las aplicaciones o productos de software cuando son lanzados al mercado siempre se estima que tengan cierto grado de aceptación entre los usuarios, para ello dependerá de las características que considere importantes el usuario. Desde el punto de vista de la Ingeniería de Software, una de las principales características que debe tener una aplicación web para que sea exitosa es que sea de calidad. Por ello resulta relevante para los desarrolladores evaluar la calidad en las aplicaciones ya que anteriormente se necesita saber qué es lo que se debe medir y cómo se debe realizar.

La calidad del software se toma en consideración de acuerdo a los atributos especificados en la norma ISO/IEC 9126 y para definirla valdría medir la lista de atributos de la calidad, en los cuales dentro de estos está la funcionalidad, fiabilidad, usabilidad, eficiencia, mantenibilidad y portabilidad.

La presente investigación plantea una evaluación de los Frameworks para el desarrollo de aplicaciones web con Python, considerando las métricas de calidad en las cuales especifica los requerimientos tanto funcionales como no funcionales para obtener un software con el menor tiempo de desarrollo.

El objetivo de la investigación es evaluar a los frameworks para el desarrollo de aplicaciones web con Python, con el fin de mejorar el desempeño de éstos, basados en métricas de calidad permitiendo al desarrollador gestionar de manera óptima el desarrollo de la aplicación.

¿Qué prestaciones trae consigo el uso de los frameworks para el desarrollo de aplicaciones web a futuras empresas desarrolladoras en la Ciudad de Machala?

H1: Los frameworks brindan las mejores prestaciones para el desarrollo de aplicaciones web en la Ciudad de Machala permitiendo obtener aplicaciones de calidad.

\section{FUNDAMENTOS TEÓRICOS}

\section{A. Lenguaje de programación}

"Un lenguaje de programación es un lenguaje que puede ser utilizado para controlar el comportamiento de una máquina, particularmente una computadora. Consiste en un conjunto de reglas sintácticas y semánticas que definen su estructura y el significado de sus elementos, respectivamente." [1]

Lenguaje diseñado que puede expresarse por medio de procesos para ser llevados a cabo a través de computadoras y que son utilizadas para la creación de programas permitiendo controlar el comportamiento físico y lógico de una máquina.

Un lenguaje de programación no son aplicaciones sino más bien herramientas de ayuda que permiten construir y adecuarlas a aplicaciones que se vayan a realizar.

\section{B. Lenguaje de programación Python}

"Python es un lenguaje de programación interpretado e interactivo, capaz de ejecutarse en una gran cantidad d plataformas. Se desarrolla como un proyecto de código abierto, administrado por PYTHON Software Foundation." [2]

Es un lenguaje de programación de código abierto que permite la ejecución en diversas plataformas, los usuarios que utilizan este lenguaje lo consideran el más elegante y a su vez 
amigable para la programación web, el principal objetivo de este lenguaje es buscar la factibilidad tanto para la lectura como el diseño, al ser un lenguaje multiparadigma brinda innumerables beneficios al permitir al usuario trabajar bajo varios estilos: programación orientada a objetos, programación funcional, entre otros. Otro aspecto importante a considerar es que permite la facilidad de extensión esto quiere decir que se puede escribir nuevos módulos de manera fácil en bajo lenguaje como $\mathrm{C}$ o $\mathrm{C}++\mathrm{y}$ se puede incluir para aplicaciones que necesiten una interfaz programable.

\section{1) Caracteristicas de Python}

TABLA I. CARACTERÍSTICAS DEL LENGUAJE DE PROGRAMACIÓN PYTHON

\begin{tabular}{|c|l|l|}
\hline \multicolumn{3}{|c|}{ CARACTERÍSTICAS } \\
\hline Legible y elegantes & Imposible escribir código ofuscado. \\
\hline Simple y poderoso & - $\begin{array}{l}\text { Soporta objetos y estructuras de datos de alto } \\
\text { nivel: strings, listas, diccionarios, etc. }\end{array}$ \\
& $\begin{array}{l}\text { Múltiples niveles de organización código: } \\
\text { funciones, clases, módulos y paquetes. } \\
\text { Incluye librerías que contiene un sinfín de } \\
\text { clases de utilidad. }\end{array}$ \\
\hline Scripting & - $\begin{array}{l}\text { No tiene que declarar constantes y variables } \\
\text { antes de utilizarlas. }\end{array}$ \\
\hline Sripting & $-\begin{array}{l}\text { No requiere paso de compilación. } \\
\text { Alta velocidad de desarrollo y buen } \\
\text { rendimiento. }\end{array}$ \\
\hline Código & $-\quad \begin{array}{l}\text { No requiere paso de compilación. } \\
\text { Alta velocidad de desarrollo y buen } \\
\text { rendimiento. }\end{array}$ \\
\hline interoperable & $-\quad \begin{array}{l}\text { Se puede utilizar en múltiples plataformas (más } \\
\text { aún que Java). } \\
\text { Ejecutar Python dentro de una JVM (Jython) }\end{array}$ \\
\hline Open Source & $-\quad \begin{array}{l}\text { Razón por la cual Python sigue creciendo y } \\
\text { creciendo. }\end{array}$ \\
\hline Propósito general & $-\quad \begin{array}{l}\text { Puedes hacer en Python todo lo que pueden } \\
\text { hacer con C\# o Java. }\end{array}$ \\
\hline
\end{tabular}

Las características antes mencionadas permitieron tener una visión de los aspectos importantes que se debe considerar del lenguaje de programación Python, considerando que es de código abierto, utilizable en múltiples plataformas, niveles de organización: código, funciones, clases, entre otras.

\section{C. ¿Qué es un framework?}

"Conjunto de conocimientos y técnicas cuya aplicación permite la utilización racional de los materiales y de los recursos naturales, mediante invenciones, construcciones $\mathrm{u}$ otras realizaciones provechosas para el hombre." [3].

Se puede definir a un framework como un armazón, que vendría a ser como una estructura el cual contiene técnicas mediante la utilización de todos los elementos que sean necesarios para beneficio del ser humano.

Python es un lenguaje considerado para tomar en cuenta en el desarrollo de aplicaciones cliente, aplicaciones web y sistemas distribuidos, mientras que no es adaptable.

\section{D. ¿Qué es un framework web?}

"'Un Framework para aplicaciones web se puede considerar como una aplicación genérica incompleta $\mathrm{y}$ configurable, con directrices arquitectónicas ofreciendo al desarrollador un conjunto de herramientas para agilitar el proceso de construir una aplicación web concreta, siempre teniendo en cuenta que es necesario adaptarlo para cada una de las aplicaciones a desarrollarse." [4]

Un framework web es una estructura que se encuentra definida que permite la reutilización de sus componentes, los cuales facilitan la creación de estas aplicaciones permitiendo ahorrar tiempo y mantenimiento.

\section{1) Objetivos}

Facilitan el desarrollo de aplicaciones web.

Permiten acelerar el proceso de desarrollo de las mismas. Reutilización de código.

\section{E. Desarrollo de aplicaciones web mediante la utilización de} frameworks

\section{1) Definición}

"En el desarrollo de software, un framework es una composición conceptual y tecnológica con un soporte bien definido, habitualmente con módulos de software concretos, en base a la cual otro proyecto de software puede ser fácilmente organizado y desarrollo." [5]

Para el desarrollo de software se hace imprescindible el uso de frameworks ya que incluyen bibliotecas, lenguaje, soportes entre otras herramientas la cuales facilitan el desarrollo de aplicaciones web.

\section{2) Arquitectura de los frameworks}

La arquitectura que poseen los frameworks interactúa bajo el Modelo Vista Controlador (MVC) ya que los desarrolladores deben dividir la documentación.

\section{a) Ventajas}

"Separación clara entre los componentes de un programa; lo cual permite su implementación por separado, la interfaz de programación de aplicaciones API (AplicationProgramming Interface) está muy bien definida; cualquiera que use el API, podrá reemplazar el Modelo, la Vista o el Controlador, sin aparente dificultad y la conexión entre el modelo y sus vistas dinámicas; se produce en tiempo de ejecución, no en tiempo de compilación." [6]

Las ventajas que posee la arquitectura es que permite la separación de sus componentes es decir la implementación se la realiza de manera separada, al igual que el API está muy bien definida ya que es clara en su uso y se produce su tiempo de ejecución dependiendo del modelo y de sus vistas.

\section{b) Modelo}

Este modelo es aquel que es realizado por el desarrollador y que contiene todos los datos, es decir toda la información y la funcionalidad del programa.

\section{c) Vista}

Una vista es aquella que permite gestionar como los datos se presentarán, es decir como interactúa el usuario final con la interfaz, la cual debe ser amigable para el cliente.

\section{d) Controlador}

En este aspecto toda la información requerida es enviada al gestor de base de datos para ser guardada, es decir controla el acceso a los datos y de esta manera el contenido es de forma estática y dinámica..

\section{F. Tipos de Frameworks}

\section{1) Django}

"Es un framework web de código abierto escrito en Python que permite construir aplicaciones web más rápido y con menos código, fue inicialmente desarrollado para gestionar aplicaciones web de páginas orientadas a noticias de World Online, más tarde se liberó bajo licencia BSD." [1]

Django es un framework para desarrollar una aplicación web gratuita de código abierto, escrito por Python el cual 
respeta el modelo vista controlador. Contiene un conjunto de componentes que permite desarrollar sitios web de manera más fácil y rápida.

\section{2) Pyramid}

Es un marco de trabajo para desarrollar aplicaciones web que tiene como características, la flexibilidad y escalabilidad. Funciona en diferentes sistemas operativos, fácil de instalar entre otras.

"Está integrado para trabajar con diferentes bases de datos, en las que se encuentra la SQLAlchemy, Zope y otros NoSQL." [7]

\section{3) Turbogear}

"TurboGears es un framework de aplicaciones web de Python, que se compone de varios módulos. Está diseñado alrededor de la arquitectura MVC que son similares a Ruby on Rails o Strut. TurboGears están diseñados para hacer que el desarrollo rápido de aplicaciones web en Python más fácil y más soportable. TurboGears es un framework de aplicaciones web escrito en Python. TurboGears sigue el paradigma Modelo Vista Controlador al igual que los marcos web más modernos como Rails, Django, puntales, etc." [8]

TurboGear es un framework enfocado en la arquitectura modelo-vista-controlador el cual está compuesto de varias fases o módulos, tiene mucha similitud a las arquitecturas de Ruby o Strut. Cabe destacar que TurboGear está diseñado con el fin de lograr un desarrollo más rápido y de buen soporte de aplicaciones web en Python.

\section{4) Web2py}

"Es un framework empresarial completo libre y de código abierto para desarrollo ágil de aplicaciones web rápidas, escalables, seguras y portables basadas en bases de datos. Escrito y programable en Python." [9]

Es decir es un marco de desarrollo integrado de código abierto para el desarrollo de aplicaciones web seguras que están conectadas a bases de datos y a su vez están programadas en Python, estos a su vez contiene todos los componentes necesarios para su desarrollo.

\section{G. Calidad del Software}

La calidad del software son aquellas características que son propias del software, el desarrollo del mismo no se lo fabrica sino más bien de desarrolla a media como incrementa la productividad en el desarrollo de aplicaciones web, considerando ciertos criterios que se debe evaluar para que un proyecto sea de calidad.

\section{H. ISO 9126}

"El estándar ISO 9126 es una norma internacional para la evaluación de software. La norma se divide en cuatro partes que aborda, respectivamente, los siguientes temas: modelo de calidad, métricas externas, métricas internas, y la calidad en el uso de métricas. La primera parte del ISO 9126, denominada ISO 9126-1 es una extensión del trabajo previo realizado por McCall (1977), Boehm (1978), FURPS y otros en la definición de un conjunto de características de calidad de software." [5]

Esta norma propone el modelo de calidad que permite evaluar la calidad de un producto, el cual se divide en cuatro aspectos importantes como son: modelo de calidad, métricas externas, métricas internas y métricas de calidad en uso.

Entre las características que posee este modelo se encuentran 6 parámetros: Funcionalidad, confiabilidad, usabilidad, eficiencia, mantenibilidad y portabilidad.

\section{1) Criterios de Calidad de Software}

Estos criterios pertenecen a la norma ISO: 9126 que abarca sobre la calidad de software en el producto final. Estos patrones de software tienen como objetivo principal mejorar la calidad de software. Existen varios patrones que son evaluados con los criterios que abarca esta norma.

TABLA II. CARACTERÍSTICAS Y SUB CARACTERÍSTICAS SEGÚN LA NORMA ISO / IEC 9126

\begin{tabular}{|c|c|c|}
\hline CARACTERÍSTICA & DEFINICIÓN & $\begin{array}{c}\text { SUB- } \\
\text { CARACTERÍSTICA }\end{array}$ \\
\hline Funcionalidad & $\begin{array}{l}\text { Busca medir los aportes } \\
\text { de un patrón de software } \\
\text { a la seguridad de acceso } \\
\text { del framework. }\end{array}$ & $\begin{array}{l}\text { - Exactitud } \\
\text { - Adecuación } \\
\text { - Interoperabilidad } \\
\text { - Seguridad }\end{array}$ \\
\hline Fiabilidad & $\begin{array}{l}\text { Calcula el aporte de los } \\
\text { patrones de software } \\
\text { para dar solución a las } \\
\text { falencias que tenga el } \\
\text { software y poder } \\
\text { recuperarse luego de } \\
\text { dichos problemas. }\end{array}$ & $\begin{array}{l}\text { - } \text { Madurez } \\
\text { - Tolerancia a fallos } \\
\text { - Recuperabilidad }\end{array}$ \\
\hline Usabilidad & $\begin{array}{l}\text { Busca cumplir con la } \\
\text { función de evaluar la } \\
\text { facilidad de utilizar un } \\
\text { patrón dado. }\end{array}$ & $\begin{array}{l}\text { - } \\
\text { - } \text { Ontendibilidad } \\
\text { - } \text { Capacididad de } \\
\text { aprendizaje }\end{array}$ \\
\hline Eficiencia & $\begin{array}{l}\text { Realiza la evaluación } \\
\text { del rendimiento de un } \\
\text { sistema con en un } \\
\text { tiempo determinado. }\end{array}$ & $\begin{array}{l}\text { - Comportamiento } \\
\text { en el tiempo } \\
\text { - Comportamiento } \\
\text { de recursos. }\end{array}$ \\
\hline Mantenibilidad & $\begin{array}{l}\text { Cumple con la función } \\
\text { de evaluar la realización } \\
\text { de correcciones, o algún } \\
\text { tipo de cambio en la } \\
\text { funcionalidad, realizado } \\
\text { bajo el patrón de } \\
\text { software evaluado. }\end{array}$ & $\begin{array}{l}\text { - } \text { Capacidad de } \\
\text { pruebas } \\
\text { - Estabilidad } \\
\text { - Modificabilidad }\end{array}$ \\
\hline Portabilidad & $\begin{array}{l}\text { Busca cumplir con la } \\
\text { función de evaluar la } \\
\text { facilidad de adaptación } \\
\text { y reemplazo. }\end{array}$ & $\begin{array}{l}\text { - Reemplazabilidad } \\
\text { - Instabilidad } \\
\text { - Adaptabilidad }\end{array}$ \\
\hline
\end{tabular}

\section{METODOLOGÍA}

Para la correspondiente investigación se emplearon los siguientes métodos de investigación:

Método analítico: se lo emplea porque permite analizar de manera minuciosa hechos en particular, en este caso se realizó el análisis tomando en consideración las características de la métrica de calidad según el estándar internacional ISO/IEC 9126 y de esta manera determinar cuál es el framework que mejor se adapta para trabajar bajo el lenguaje Python para obtener aplicaciones web de calidad.

Método descriptivo: se lo emplea al describir las prestaciones, tecnologías, herramientas y librerías que cada uno de los Frameworks posee tomando en cuenta cada una de las características según la métrica de calidad del estándar ISO/IEC 9126, para posteriormente realizar un análisis exhaustivo de los resultados, para de esta manera emitir criterios que sean de ayudan y enriquezcan el conocimiento.

Método bibliográfico: se lo emplea en la búsqueda de información sobre cuestiones determinadas que en este caso es evaluar a los frameworks para obtener aplicaciones de calidad, buscando en fuentes confiables ya que es indispensable para el progreso de la investigación.

Tomando en cuenta todos estos métodos, se analiza mediante la norma ISO/IEC 9126, ya que es un estándar internacional para evaluar la calidad de los frameworks; esta norma consta de seis características, las cuales permiten 
determinar cuál es el mejor marco de trabajo para el desarrollo de aplicaciones web.

\section{Definición de indicadores}

\section{1) Características y sub-características de evaluación}

Las características y sub-características que se evaluará y que están inmersos en la evaluación de los frameworks se los tomó del estándar internacional ISO/IEC 9621 de la calidad del software las cuales son:

Funcionalidad: capacidad de los frameworks de proveer los servicios necesarios para que cumpla con los requerimientos funcionales.

La funcionalidad trata en si en como los frameworks cumpla adecuadamente con las necesidades para las cuales fue diseñado o se lo utiliza.

Fiabilidad: capacidad de los frameworks para suministrar los servicios necesarios para efectuar con los requerimientos funcionales.

En la fiabilidad se toma en cuenta la tolerancia a fallos, la manera de que los frameworks puedan operar libre de fallos durante un tiempo y entorno determinado, es decir, la probabilidad de hasta dónde puede los frameworks llevar a cabo su función con la exactitud requerida.

En la calidad del software cualquier tipo de falla sin importar su significancia, afecta la sincronía con respecto a los requisitos y requerimientos del software; la fiabilidad es una de las métricas más influyentes en el momento de medir o hablar de calidad.

Usabilidad: esfuerzo requerido por el usuario para utilizar el producto satisfactoriamente.

La usabilidad juega un papel importante debido a que va ligada a la calidad que debe tener un framework. Basado en el estándar internacional ISO/IEC 9126 se puede definir a la usabilidad como "el grado en el que un framework es utilizado por los desarrolladores para conseguir los objetivos específicos con efectividad, eficiencia y satisfacción en un determinado contexto. Lo que significa que este estándar relaciona a los frameworks con el uso que le dan los desarrolladores para alcanzar su satisfacción. Las aplicaciones web deben estar construidas para alcanzar la usabilidad, el cual toma en cuenta algunos atributos que permite evaluar el esfuerzo necesario que el desarrollador deberá invertir para que el usuario utilice la aplicación.

Eficiencia: relación entre las prestaciones del software y los requisitos necesarios para su utilización.

Cuando se trata de desarrollo de aplicaciones web una de las métricas más comunes que se mencionan es la eficiencia, lo cual se cae en una confusión con la eficacia; la eficiencia es la habilidad o capacidad que tiene el framework para mantener prestaciones apropiadas relativas al uso sobre los recursos que se deba tomar en cuenta, es decir los frameworks son eficientes cuando cumple con las necesidades que tenga el desarrollador usando lo mínimo de recursos posibles que le son dispuestos.

Mantenibilidad: esfuerzo necesario para adaptarse a las nuevas especificaciones y requisitos del software.

Esta característica se la define como la capacidad de un framework para ser modificado, dichas modificaciones pueden incluir correcciones, mejoras o que sea adaptable a nuevos entornos de trabajo, es decir, la mantenibilidad se refiere a los atributos que permiten medir el esfuerzo necesario para realizar modificaciones en el mismo, ya sea por corrección de errores o por el incremento de funcionalidad.

Portabilidad: capacidad del software ser transferido de un entorno a otro.
Se define a la portabilidad como la capacidad que tiene un framework para ser adaptado desde un entorno a otro, esto quiere decir que sin importar el lenguaje de programación el framework se acople a las funciones de otro lenguaje y no sufra transformaciones o fallos al ser utilizado.

\section{2) Implementación de criterios de evaluación}

Para evaluar a los frameworks tomando en cuenta a las características de la norma ISO/IEC 9126 se hace uso de la escala de Likert, teniendo como propósito el de cuantificar la calidad tomando en cuenta las características antes mencionadas y así determinar cuál es el marco de trabajo más factible para su uso. A continuación, las escalas de evaluación:

TABLA III. ESCALA DE LIKERT

\begin{tabular}{|c|c|}
\hline \multicolumn{2}{|c|}{ Criterio de evaluación } \\
\hline Valor & Valor \\
\hline 1 & 1 \\
\hline 2 & 2 \\
\hline 3 & 3 \\
\hline 4 & 4 \\
\hline 5 & 5 \\
\hline
\end{tabular}

Selección de un área piloto de estudio

El área de estudio en donde se fundamenta la investigación a la cual se la considera como la apropiada para el estudio respectivo de la "Evaluación de los frameworks de Python" fueron todas las empresas desarrolladoras de software de la Ciudad de Machala.

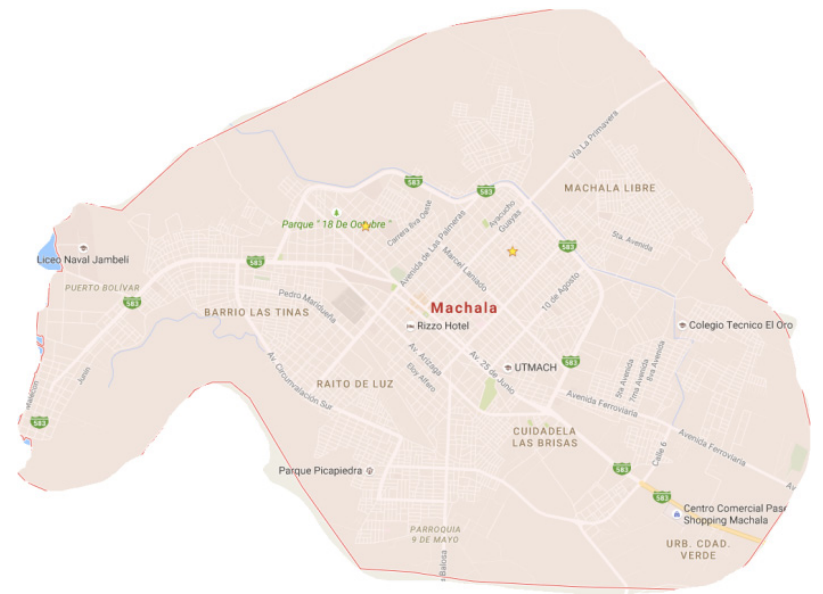

Fig. 1. Ciudad piloto para el estudio respectivo

Según la información originada por las autoridades del Servicio de Rentas Internas, esta ciudad cuenta con cinco empresas activas en su totalidad. Las cuales son las siguientes:

TABLA IV. EMPRESAS DESARROLLADORAS DE SOFTWARE

\begin{tabular}{|c|c|}
\hline $\mathbf{N}^{\mathbf{0}}$ & $\begin{array}{c}\text { Empresas desarrolladoras de software } \\
\text { en la ciudad de Machala }\end{array}$ \\
\hline $\mathbf{1}$ & Ecuasis \\
\hline $\mathbf{2}$ & Ole Sistemas \\
\hline $\mathbf{3}$ & SofAdcon \\
\hline $\mathbf{4}$ & Pagupa Soft \\
\hline $\mathbf{5}$ & Ofsercont \\
\hline
\end{tabular}

\section{MATERIALES Y MÉTODOS}

Para la comprobación de la hipótesis planteada será necesaria la aplicación de un método que permitirá establecer una secuencia ordenada de acciones que llevará a establecer las 
conclusiones sobre el uso del mejor framework de calidad para el desarrollo de aplicaciones web.

Para obtener información pertinente y necesaria para la investigación se recolectaron datos, los cuales se los obtuvo mediante el desarrollado encuestas y entrevistas a los desarrolladores encargados de las diferentes organizaciones, a quiénes se les aplicó las preguntas de las encuestas planteadas. En las encuestas que se formularon se establecieron una serie de preguntas las cuales estaban basadas en los Frameworks que utilizan las empresas para el desarrollo de aplicaciones web tomando en consideración al estándar internacional ISO/IEC 9126 para lo cual se obtuvo sus resultados mediante criterios de evaluación para de esta manera estimar las características planteadas por la métrica de calidad.

Para una mejor comprensión de los resultados obtenidos en base a las encuestas formuladas, e las planteó mediante tablas y gráficos estadísticos donde se representó la información recopilada consiguiendo así una mejor interpretación de los mismos.

Por último, para evaluar la hipótesis se tomó en consideración al framework con mayor puntaje, el cual resultó de la suma de los indicadores pre-establecidos.

\section{RESUltados}

Mediante la recopilación de información que se obtuvo de encuestas realizadas a empresas desarrolladoras de software aplicadas a la Ciudad de Machala, se pudo determinar cuál es el framework de calidad que mejor se adopte para el desarrollo de aplicaciones web bajo el lenguaje Python.

En el proceso de la investigación obtuvo como resultado que la población de estudio fue de 5 empresas, de las cuales 2 determinaron que utilizan frameworks para el desarrollo de aplicaciones web con lenguaje de programación Python y 3 de JAVA.

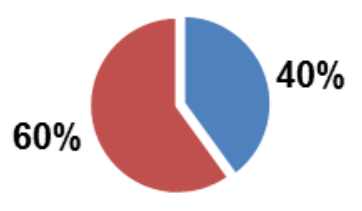

- Python - JAVA

Fig. 2. Utilización de frameworks en las empresas

El $60 \%$ de empresas utilizan frameworks con el lenguaje de programación JAVA y el $40 \%$ con Python, lo que equivale a 3 y 2 empresas respectivamente.

A continuación, se presentan los resultados de las encuestas realizadas a las empresas desarrolladoras en la que se tomó en cuenta las características de la norma ISO/IEC 9126 para evaluar al mejor framework de calidad para el desarrollo de aplicaciones web.

Datos respecto a los frameworks

TABLA V. EMPRESAS Y SUS ABREVIATURAS

\begin{tabular}{|c|c|}
\hline \multicolumn{2}{|c|}{ EMPRESAS Y ABREVIATURAS } \\
\hline Abreviatura & Definición \\
\hline E1 & Empresa 1 (Ole Sistemas) \\
\hline E2 & Empresa 2 (Ofsercont) \\
\hline
\end{tabular}

1) Evaluación de los frameworks mediante las características de la métrica de calidad de la norma ISO/IEC 9126

\section{a) Functionality}

TABLA VI. FUNCIONALIDAD DE LOS FRAMEWORKS MÁS UTILIZADOS

\begin{tabular}{|c|c|c|c|c|c|c|c|c|c|c|c|c|}
\hline \multicolumn{13}{|c|}{ FUNCIONALIDAD } \\
\hline & \multicolumn{3}{|c|}{ DJANGO } & \multicolumn{3}{|c|}{$\begin{array}{l}\text { PYRAMID } \\
\end{array}$} & \multicolumn{3}{|c|}{ TURBOGEAR } & \multicolumn{3}{|c|}{ WEB2PY } \\
\hline MÉTRICAS & ш & జี & $\frac{\overrightarrow{5}}{\frac{1}{2}}$ & 亗 & జี & $\frac{\overrightarrow{1}}{0}$ & ш & జี & $\frac{\vec{\pi}}{\frac{1}{2}}$ & 岀 & జิ & $\frac{\overrightarrow{1}}{5}$ \\
\hline EXACTITUD & 5 & 5 & 10 & 5 & 1 & 6 & 5 & 3 & 8 & 5 & 4 & 9 \\
\hline $\begin{array}{l}\text { ADECUA- } \\
\text { CION }\end{array}$ & 3 & 2 & 5 & 3 & 2 & 5 & 3 & 2 & 5 & 3 & 2 & 5 \\
\hline $\begin{array}{l}\text { INTEROPE- } \\
\text { RABILIDAD }\end{array}$ & 2 & 3 & 5 & 2 & 1 & 3 & 2 & 2 & 4 & 2 & 2 & 4 \\
\hline SEGURIDAD & 4 & 2 & 6 & 4 & 2 & 6 & 4 & 1 & 5 & 4 & 2 & 6 \\
\hline TOTAL & & 6.5 & & & 5 & & & 5.5 & & & 6 & \\
\hline
\end{tabular}

Se puede observar que, entre los 4 frameworks, sobresale Django en la métrica de funcionalidad y se puede evidenciar porque permite realizar las tareas de manera eficiente y a la vez evalúa el resultado final verificando que tenga consistencia lo que el usuario desea hacer en él y que permita la interacción con otro sistema, es decir la interoperabilidad con otro lenguaje.

\section{b) Fiabilidad}

TABLA VII: FIABILIDAD DE LOS FRAMEWORKS MÁS UTILIZADOS

\begin{tabular}{|c|c|c|c|c|c|c|c|c|c|c|c|c|}
\hline \multicolumn{13}{|c|}{ FIABILIDAD } \\
\hline & \multicolumn{3}{|c|}{ DJANGO } & \multicolumn{3}{|c|}{ PYRAMID } & \multicolumn{3}{|c|}{ TURBOGEAR } & \multicolumn{3}{|c|}{ WEB2PY } \\
\hline MÉTRICAS & 岀 & พิ & $\frac{\vec{K}}{0}$ & ш & జี & $\frac{\vec{E}}{0}$ & 岀 & జิ & $\begin{array}{l}\vec{E} \\
\text { 음 }\end{array}$ & 出 & พี & 胥 \\
\hline MADUREZ & 5 & 5 & 10 & 5 & 1 & 6 & 5 & 3 & 8 & 5 & 4 & 9 \\
\hline $\begin{array}{l}\text { TOLERANCIA A } \\
\text { FALLOS }\end{array}$ & 3 & 2 & 5 & 1 & 2 & 3 & 4 & 2 & 6 & 3 & 1 & 4 \\
\hline RECUPERABILIDAD & 2 & 4 & 6 & 2 & 1 & 3 & 2 & 1 & 3 & 1 & 2 & 3 \\
\hline TOTAL & & 7 & & & 4 & & & 5.66 & & & 5.33 & \\
\hline
\end{tabular}

Se puede observar que la diferencia es muy notable, Django es una herramienta propietaria que garantiza a sus desarrolladores un soporte a cualquier fallo en el sistema, además de la recuperabilidad de algún fallo que ocurra por motivo de haberse cerrado de manera inesperada ya que permite tener de nuevo lo que se haya tenido hecho.

\section{c) Usabilidad}

TABLA VIII. USABILIDAD DE LOS FRAMEWORKS MÁS UTILIZADOS.

\begin{tabular}{|c|c|c|c|c|c|c|c|c|c|c|c|c|}
\hline \multicolumn{13}{|c|}{ USABILIDAD } \\
\hline & \multicolumn{3}{|c|}{ DJANGO } & \multicolumn{3}{|c|}{ PYRAMID } & \multicolumn{3}{|c|}{ TURBOGEAR } & \multicolumn{3}{|c|}{ WEB2PY } \\
\hline MÉTRICA & ш & జิ & $\underset{2}{\vec{E}}$ & w & พิ & 壱 & 出 & జี & $\frac{1}{2}$ & एu & พี & $\begin{array}{l}\vec{E} \\
0 \\
0\end{array}$ \\
\hline ENTENDIBILIDAD & 3 & 6 & 9 & 3 & 2 & 5 & 2 & 2 & 4 & 3 & 4 & 7 \\
\hline OPERABILIDAD & 4 & 4 & 8 & 3 & 2 & 5 & 4 & 1 & 5 & 3 & 3 & 6 \\
\hline $\begin{array}{l}\text { CAPACIDAD DE } \\
\text { APRENDIZAJE }\end{array}$ & 2 & 4 & 6 & 3 & 1 & 4 & 2 & 1 & 3 & 2 & 3 & 5 \\
\hline TOTAL & \multicolumn{3}{|c|}{7.66} & \multicolumn{3}{|c|}{4.66} & \multicolumn{3}{|c|}{4} & \multicolumn{3}{|c|}{6} \\
\hline
\end{tabular}

Se puede observar que continúa predominando el framework Django, lo que resulta visible que es una herramienta muy usado por los desarrolladores ya que su interfaz es amigable para el usuario y se puede adaptar a las necesidades que se tenga para de esta manera aprovecharla al máximo según sean los requerimientos del desarrollador para la creación de aplicaciones. 


\section{d) Eficiencia}

TABLA IX: EFICIENCIA DE LOS FRAMEWORKS MÁS UTILIZADOS

\begin{tabular}{|c|c|c|c|c|c|c|c|c|c|c|c|c|}
\hline \multicolumn{13}{|c|}{ EFICIENCIA } \\
\hline \multirow[b]{2}{*}{ MÉTRICA } & \multicolumn{3}{|c|}{ DJANGO } & \multicolumn{3}{|c|}{ PYRAMID } & \multicolumn{3}{|c|}{ TURBOGEAR } & \multicolumn{3}{|c|}{ WEB2PY } \\
\hline & ш & พี & $\begin{array}{l}\vec{E} \\
0\end{array}$ & ш & พี & $\frac{\vec{T}}{0}$ & ш & พี & $\begin{array}{l}\vec{E} \\
0\end{array}$ & 出 & జิ & $\frac{\vec{K}}{0}$ \\
\hline $\begin{array}{l}\text { COMPORTAMIENTO } \\
\text { EN EL TIEMPO }\end{array}$ & 5 & 4 & 9 & 3 & 2 & 5 & 2 & 2 & 4 & 3 & 5 & 8 \\
\hline $\begin{array}{l}\text { COMPORTAMIENTO } \\
\text { DE RECURSOS }\end{array}$ & 4 & 4 & 8 & 3 & 1 & 4 & 4 & 1 & 5 & 3 & 5 & 8 \\
\hline TOTAL & & 8.5 & & & 4.5 & & & 4.5 & & & 8 & \\
\hline
\end{tabular}

Se puede observar que el framework Django tiene un grado de aceptación inigualable en comparación con los otros, puesto que es el más completo para la realización de aplicaciones, aunque no existe mucha diferencia con el Web2PY, ya que este también posee una gama de recursos disponibles en comparación con el Django, este framework verifica la rapidez con que responde mediante la utilización para desarrollar aplicaciones al igual que determina que los recursos que se utilicen sean aprovechables de manera eficiente.

\section{e) Mantenibilidad}

TABLA X. MANTENIBILIDAD DE LOS FRAMEWORKS MÁS UTILIZADOS

\begin{tabular}{|c|c|c|c|c|c|c|c|c|c|c|c|c|}
\hline \multicolumn{13}{|c|}{ MANTENIBILIDAD } \\
\hline & \multicolumn{3}{|c|}{ DJANGO } & \multicolumn{3}{|c|}{ PYRAMID } & \multicolumn{3}{|c|}{ TURBOGEAR } & \multicolumn{3}{|c|}{ WEB2PY } \\
\hline MÉTRICA & w & జ & $\frac{\vec{E}}{\circ}$ & ш & జี & 咅 & $\bar{\omega}$ & జี & $\frac{5}{5}$ & w & జు & $\frac{\vec{E}}{\circ}$ \\
\hline $\begin{array}{l}\text { CAPACIDAD DE } \\
\text { PRUEBAS }\end{array}$ & 5 & 4 & 9 & 2 & 4 & 6 & 5 & 1 & 6 & 5 & 3 & 8 \\
\hline ESTABILIDAD & 3 & 4 & 7 & 1 & 2 & 3 & 4 & 2 & 6 & 3 & 1 & 4 \\
\hline $\begin{array}{l}\text { MODIFICA- } \\
\text { BILIDAD }\end{array}$ & 2 & 4 & 6 & 2 & 2 & 4 & 2 & 1 & 3 & 3 & 2 & 5 \\
\hline TOTAL & \multicolumn{3}{|c|}{7.33} & \multicolumn{3}{|c|}{4.33} & \multicolumn{3}{|c|}{5} & \multicolumn{3}{|c|}{5.66} \\
\hline
\end{tabular}

Se puede observar que el framework Django es más efectivo, gracias al soporte de mantenibilidad que posee debido ya que permite verificar que el marco de trabajo continúe funcionando a pesar de estar inmerso a cambios, al igual que evalúa que su manejo este probado y modificable de manera fácil.

\section{f) Portabilidad}

TABLA XI. PORTABILIDAD DE LOS FRAMEWORKS MÁS UTILIZADOS

\begin{tabular}{|c|c|c|c|c|c|c|c|c|c|c|c|c|}
\hline \multicolumn{13}{|c|}{ PORTABILIDAD } \\
\hline & \multicolumn{3}{|c|}{ DJANGO } & \multicolumn{3}{|c|}{ PYRAMID } & \multicolumn{3}{|c|}{ TURBOGEAR } & \multicolumn{3}{|c|}{ WEB2PY } \\
\hline METRICS & $\bar{w}$ & జు & 站 & 岀 & జิ & 壳 & 出 & $\widetilde{\Psi}$ & 苫 & 出 & $\widetilde{w}$ & 咅 \\
\hline REPLACEABILITY & 5 & 5 & 10 & 4 & 4 & 8 & 5 & 3 & 8 & 5 & 5 & 10 \\
\hline STABILITY & 5 & 5 & 10 & 4 & 4 & 8 & 5 & 3 & 8 & 5 & 4 & 9 \\
\hline ADAPTABILITY & 5 & 5 & 10 & 5 & 3 & 8 & 4 & 3 & 7 & 5 & 4 & 9 \\
\hline TOTAL & & 10 & & & 8 & & & 9 & & & 9.33 & \\
\hline
\end{tabular}

Se puede observar que los resultados arrojaron que todos los frameworks tiene un índice muy elevado en la portabilidad ya que permiten ser reemplazados por otro lenguaje, al igual que fácil de instalar y adaptables a otros ambientes.

Se puede demostrar la ventaja que tiene el framework Django en la mayoría de las características de las métricas de calidad aplicadas, dando a entender que es el mejor que se adapta a los desarrolladores para trabajar en el desarrollo de aplicaciones web.

Evaluación de los frameworks de acuerdo al estándar internacional ISO / IEC 9126
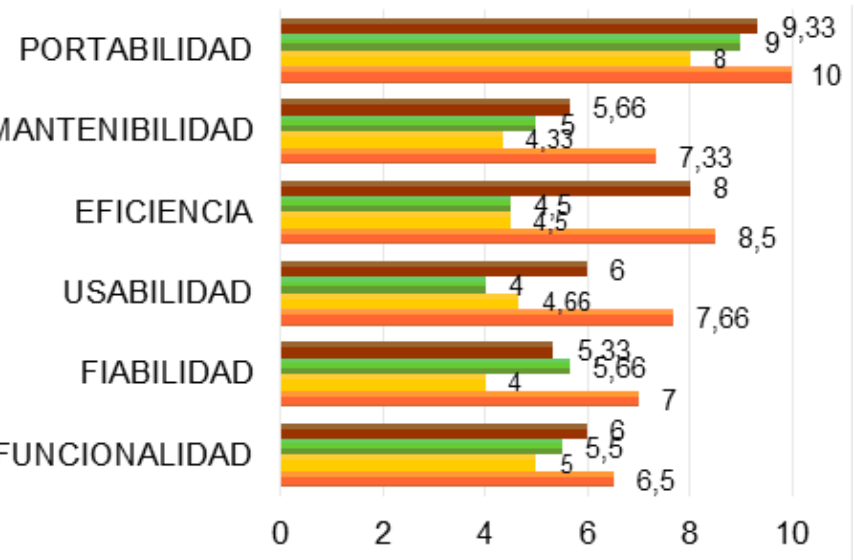

\section{-WEB2PY $=$ TURBOGEAR $\backsim$ PYRAMID $\approx$ DJANGO}

Fig. 3. Evaluación de los frameworks de acuerdo a las características de la métrica de calidad.

\section{DISCUSIÓN}

Los frameworks son indudablemente importantes en el desarrollo de aplicaciones web, ya que facilita el trabajo a los arquitectos de software permitiendo acelerar el proceso de desarrollo mediante la reutilización de código ya existente al igual que promueve buenas prácticas de desarrollo como el uso de patrones.

Mediante la aplicación de las encuestas se ha podido evidenciar que las empresas que desarrollan aplicaciones web en la Ciudad de Machala, solo dos de ellas trabajan con el lenguaje Python, considerando como mejor framework Django.

De los resultados obtenidos se puede indicar que Django es el framework que mejor cumple con las características del modelo de calidad, es uno de los frameworks más populares y usados en el mercado de frameworks web con Python, ya que tiene la ventaja de que a partir de algunos de los módulos que incluye, permite ser integrable a muchos frameworks para el acceso a datos, además permitiendo configurar sus controles y objetos lo que hace fácilmente integrable con otros lenguajes y moldearlos para beneficio de los arquitectos de software.

Según el criterio evaluado mediante las características de la métrica de calidad que toma en cuenta la funcionalidad, fiabilidad, usabilidad, eficiencia, mantenibilidad y portabilidad permitió determinar que el framework Django cumple a cabalidad todas aquellas características nombradas anteriormente por lo tanto es el que mejor se adapta para el desarrollo de aplicaciones web que cumpla con las necesidades de los arquitectos de software.

A través de los resultados obtenidos en base a encuestas realizadas a empresas desarrolladoras de aplicaciones web de la Ciudad de Machala se aprueba la hipótesis planteada, considerando al framework Django como el mejor que se adapta para trabajar en el desarrollo de sistemas web bajo el lenguaje Python.

\section{CONCLUSIONES}

En la presente investigación se evidencia que el modelo de calidad de la norma ISO/IEC 9126 se adecua de forma correcta a la evaluación de frameworks, determinando como herramienta importante que permita garantizar cuál es el framework más conveniente que el arquitecto de software deba utilizar para el desarrollo de aplicaciones web.

Un proceso fundamental para la aplicación de un modelo de calidad es la asignación de valores de importancia que se le otorga a cada una de las características y sub-características, lo cual determina que características son más relevantes al elegir 
un framework. Sin la asignación de estos valores que se les otorga mediante encuestas realizadas resulta imposible determinar en cual se desenvuelven bien cada Framework seleccionado.

Determinando de esta manera que el Framework Django cumple de la mejor manera con las características, denotando una funcionalidad, fiabilidad, usabilidad, eficiencia, mantenibilidad y portabilidad al resto de Frameworks evaluados.

El Framework Django es una buena opción para la construcción de aplicaciones web ya que luego de la aplicación del modelo de calidad, se observa que mantiene buenas puntuaciones en pareas importantes las cuales el desarrollador debe tomar en cuenta como son la funcionalidad y usabilidad para desarrollar aplicaciones web de calidad.

\section{REFERENCIAS}

[1] B. A. B. P. Paul David Cumba Armijos, «Análisis de python con django frente a ruby on rails para desarrollo ágil de aplicaciones web. Caso práctica: Dech,» Riobamba, 2012.

[2] G. I. B. G. Carlos Patricio Chavez Ñaunay, «Estudio comparativo de las tecnologías Python y Perl para desarrollar aplicaciones web implementando al programa de alfabetización del Consejo Provincial de Chimborazo,» Riobamba, 2011.

[3] M. Degiovannini, «Comparativa de Frameworks Web,» JavaHispano.com, vol. II, p. 3, 2011.

[4] C. G. R. R. Manuel Mirana Chinlli, «Repositorio Institucional de la Escuela Superior Politécnica de Chimborazo,» 2015. [En línea]. Available: http://dspace.espoch.edu.ec/handle/123456789 14581. [Último acceso: 08 Agosto 2016].

[5] Á. P. C. R. José Luis Manzano Zumba, «Análisis comparativo de framework software libre para el desarrollo de aplicaciones de escritorio en java.,» Riobamba, 2014.

[6] Y. D. G. Yenisleidy Fernández Romero, «Patrón Modelo - Vista - Controlador,» Telematica, vol. 11, nº 1, p. 11, 2012.

[7] pylonsproject, «About Pyramid,» [En línea]. Available: http://www.pylonsproject.org/projects/pyramid/about. [Último acceso: Agosto 2016].

[8] TutorialsPoint, «TurboGear,» 17 Febrero 2016. [En línea]. Available: http://www.tutorialspoint.com/turbogears/turbogears _tutorial.pdf. [Último acceso: Agosto 2016].

[9] Web2Py, «Descargas de Web2Py,» [En línea]. Available: http://www.web2py.com.ar/examples/default/download. [Último acceso: Agosto 2016].

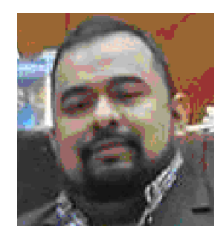

Jimmy Rolando Molina Ríos, nació en Machala - Ecuador, el 14 de Septiembre de 1984; Ingeniero de Sistemas de la Universidad Técnica de Machala en 2009; Magíster en Docencia y Gerencia en Educación Superior en 2014; Actualmente es docente investigador de la Carrera de Ingeniería de Sistemas de la Universidad Técnica de Machala.

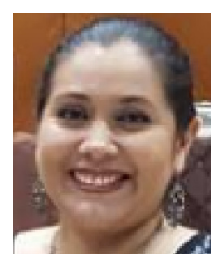

Mariuxi Paola Zea Ordóñez, nació en Pasaje - Ecuador, el 1 de Enero de 1974; Ingeniero en Computación y Ciencias de la Informática en la Escuela Politécnica Javeriana del Ecuador en 2003; Magíster en Educación Superior con mención en temas contemporáneos en docencia superior en la Universidad Casa Grande en 2011; Actualmente es docente investigador de la Carrera de Ingeniería de Sistemas de la Universidad Técnica de Machala.

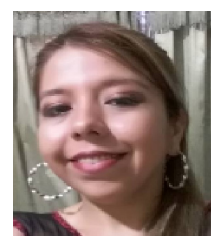

Nancy Magaly Loja Mora, nació en Zaruma - Ecuador, el 28 de Mayo de 1978; Ingeniero en Sistemas Informáticos y Computación en la Universidad Técnica Particular de Loja en 2005; Magíster en Docencia y Gerencia en Educación Superior en la Universidad de Guayaquil en 2012; Actualmente es docente investigador de la Carrera de Ingeniería de Sistemas de la Universidad Técnica de Machala.

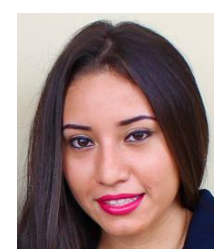

Erika Lizbeth Loaiza Sojos, nació en Machala Ecuador, el 5 de Noviembre de 1994; cursa el sexto semestre de la carrera de Ingeniería de Sistemas de la Universidad Técnica de Machala; estudiante investigadora. 